\title{
Research and Development of 2-frequency (110/138 GHz) FADIS for JT-60SA ECHCD system
}

\author{
H. Idei ${ }^{1}$, S. Moriyama ${ }^{2}$, T. Kobayashi ${ }^{2}$, A. Isayama ${ }^{2}$, M. Sakaguchi ${ }^{3}$, and W. Kasparek ${ }^{4}$ \\ ${ }^{1}$ Research Institute for Applied Mechanics, Kyushu Univ., Kasuga, 816-8580, Japan \\ ${ }^{2}$ Japan Atomic Energy Agency, Naka, 311-0193, Japan \\ ${ }^{3}$ Furukawa C \& B Co., Ltd., Yamato, 242-0018, Japan \\ ${ }^{4}$ Institute of Interfacial Process Engineering and Plasma Technology, Pfaffenwaldring 31, D-70569 Stuttgart, Germany
}

\begin{abstract}
A FAst DIrectional Switch (FADIS) of 2-frequency (2- $f$ ) gyrotron system for the JT-60SA project is being developed under collaboration between Japan Atomic Energy Agency (JAEA) and Kyushu University. At first, the frequency drift and dip in the gyrotron operation were measured to consider which kind of FADIS is preferred for application in the Electron Cyclotron Heating and Current Drive (ECHCD) system for the JT60SA. Various types of the FADIS have been considered. A square corrugated waveguide diplexer system with double resonant rings was considered as one of the most attractive FADIS systems for stable high-power and long-pulse operations in the 2- $f$ JT-60SA ECHCD system.
\end{abstract}

\section{Introduction}

Local current generation by Electron Cyclotron Current Drive (ECCD) has been used to suppress Neoclassical Tearing Mode (NTM) activities in many tokamaks such as the JT-60U [1-3], the ASDEX-U [4], the DIII-D [5]. In the JT-60U experiments, active NTM suppression was demonstrated by local ECCD with several stabilization techniques such as real-time steering of the launcher mirror [1] and preemptive stabilization [2]. Synchronous switched injection for mode rotation of the NTM magnetic island has been proposed as a more effective suppression method relative to the continuous injection. The synchronous injection experiments were conducted with different phasing between the mode rotation and the modulated ECCD injection in the JT-60U [3]. Perturbed magnetic field components resulting from the NTM activity began to be decreased by the synchronous injection in in-phase experiments, while they were not changed so much and were conversely increased by ECCD injection being out of phase by 90 and 180 degrees for the mode rotation, respectively. In the in-phase experiment, the incident power was modulated at $80 \%$, following the $100 \%$ modulated injection. The perturbed field component was slightly increased in the $80 \%$ modulated injection, but was still low level, indicating the $80 \%$ modulation was still effective for the NTM suppression [2]. The synchronous in-phase injection was effective for the NTM suppression also in experiments at ASDEX-U [4]. Up to now, the synchronous injection was based on incident power modulation of high power mm-wave tube (gyrotron). The power modulation has the disadvantage that half of the available power is out of use. An attractive alternative of the synchronous injection is switching of the launchers, i.e. directing the incident beam to different poloidal and/or toroidal ECCD positions. A FAst DIrectional Switch (FADIS) system has been proposed and developed to switch fast the transmission lines and the launchers of the ECHCD injection [6]. The JT-60SA research plan has been considered and proposed as the Broader Approach (BA) researches. The 2frequency $(2-f)$ [110/138 GHz] gyrotron has been developed for the JT-60SA project [7]. As baseline scenarios at a toroidal magnetic field of $B_{\mathrm{t}}=1.7 \mathrm{~T}$, ECHCD experiments have been considered with the $110 \mathrm{GHz}$ injection. The $138 \mathrm{GHz}$ injection has been also proposed for power deposition in the central plasma region at $B_{\mathrm{t}}=2.25 \mathrm{~T}$ [8]. Combination of operating frequencies of $110 \mathrm{GHz}$ and 138 $\mathrm{GHz}$ has been selected with oscillation modes of $\mathrm{TE}_{22,8}$ and $\mathrm{TE}_{27,10}$ in the JT-60SA gyrotron. This gyrotron uses and output diamond window with a thickness of $2.3 \mathrm{~mm}$, which corresponds to 4 and 5 times the half wavelength of 110 and $138 \mathrm{GHz}$, respectively. A collaboration task on the FADIS for the 2- $f$ gyrotron system has begun between Japan Atomic Energy Agency and Kyushu University for the effective NTM suppression in various experimental scenarios on the JT-60SA.

This paper is organized as follows. Section 2 describes frequency measurements of the JT-60SA gyrotron. Note that the FADIS operation is based on steep slopes in the diplexer transmission function leading to switching upon a small frequency shift. The gyrotron frequency can be controlled by the gun-anode or the gun-collector voltages, but it is drifting due to thermal expansion of the gyrotron cavity in general. A frequency dip has been also observed in several gyrotrons [6]. The frequency measurement is important to consider the switching property in the FADIS 


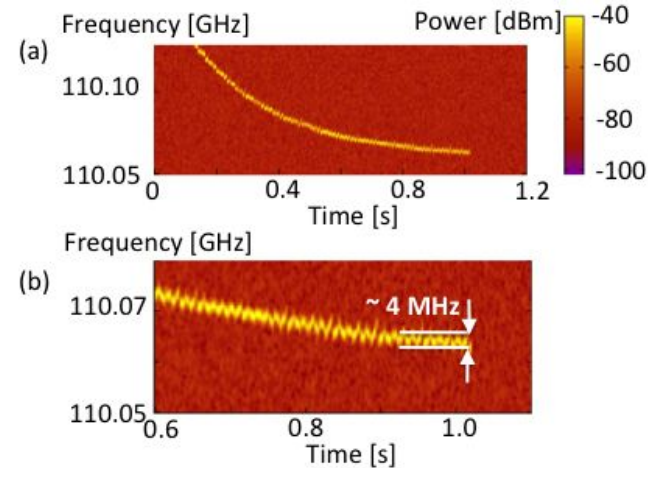

Figure 1. Time evolution of oscillating frequency measured at heterodyne detection of JT-60SA gyrotron. The frequency drifts about $100 \mathrm{MHz}$ for $1 \mathrm{sec}$ as shown in (a). Frequent dips of $\Delta f \sim$ $4 \mathrm{MHz}( \pm 2 \mathrm{MHz})$ are observed in (b).

operation. Various types of the FADIS have been considered under the measured frequency dip and controllability in the JT-60SA ECHCD system. Section 3 introduces the Mach-Zehnder interferometer and the ring resonator types in circular corrugated waveguide lines. A quasi-optical resonator ring type is introduced in Section 4, and a ring resonator with beam splitters of square corrugated waveguide is proposed for the 2- $f$ application in Section 5. Finally, the conclusion in given in Section 6.

\section{Frequency Drift and Dip of JT-60SA Gyrotron}

Oscillating frequency of the JT-60SA gyrotron was measured by higher (10-th) harmonic heterodyne detection with a Local Oscillator (LO) source, microwave synthesizer. The oscillating mm-wave RF component at the heterodyne detection was picked up at a directional coupler installed to the reflector of a miter-bend. The Intermediate Frequency (IF) component of $\sim 1 \mathrm{GHz}$ was sampled with a fast digitizer of $5 \mathrm{Gs} / \mathrm{s}$. The oscillating frequency was analyzed by Fast Fourier Transform (FFT) of the digitized IF component. Figure 1 shows time evolution of the oscillating RF frequency $(\sim 110 \mathrm{GHz})$ by combining the FFTed IF frequency with the 10-th harmonic frequency of the LO frequency $(10.9 \mathrm{GHz})$ at the $0.5 \mathrm{MW}$ output power level. The frequency drifted about $100 \mathrm{MHz}$ for $1 \mathrm{sec}$ and became stable, but with frequent dips of $\Delta f \sim 4 \mathrm{MHz}( \pm 2$ $\mathrm{MHz}$ ). These frequency dips may affect the diplexer operation based on frequency control. Active mirror-motion control systems have been developed to adjust round-trip length of the resonant ring to compensate the frequency drift and dip [9]. However, our first design target of FADIS operation is limited to a switching performance where more than $80 \%$ synchronous power is transmitted into the selected launcher with no active control. Figure 2 shows frequency control by variation of the gun-anode voltage of $\Delta V_{\mathrm{a}}=1.2 \mathrm{kV}$. A frequency change $10 \mathrm{MHz}$ was obtained. The anode voltage control system has been developed in the JT-60U ECHCD system to modulate the oscillating power [10]. The applied anode voltage extracts

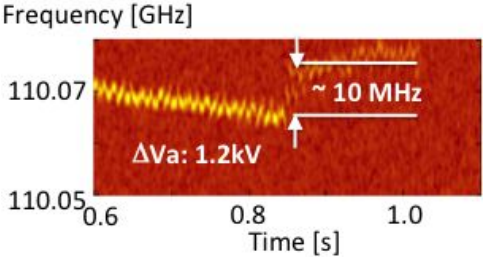

Figure 2. Frequency control by increasing gun-anode voltage of $\Delta V_{\mathrm{a}}=1.2 \mathrm{kV}$. Color mapping in $\mathrm{dBm}$ is the same as Fig.1.

Table 1. Design conditions and targets of FADIS for JT-60SA ECHCD system

\begin{tabular}{ll}
\hline Subject & Parameter \\
\hline Operating Frequency & $110 / 138 \mathrm{GHz}$ \\
Operating polarization & elliptical, if possible \\
Frequency dip & $4 \mathrm{MHz}$ \\
Switching frequency & $10 \mathrm{MHz}$ \\
Synchronous modulation rate & $>80 \%$ \\
\hline
\end{tabular}

the electron beam from the gyrotron gun, and increases the beam current in gyrotrons with a triode-type magnetron injection gun. The oscillating frequency is slightly changed together with the beam energy by a relativistic effect on electron mass. In the JT-60U/SA gyrotrons, high power/efficiency oscillations have been attained in hardexcitation regions where parameter areas of the anode voltage and the cavity magnetic filed were limited in extent for regular operations with the high power output. The high power main oscillating $\mathrm{TE}_{22,6}$ mode disappeared in the anode voltage control of $\Delta V_{\mathrm{a}} \sim 2 \mathrm{kV}$, and the oscillating mode switched to the low power $\mathrm{TE}_{21,6}$ mode in the JT-60U gyrotron [10]. This means that frequency controllability in the diplexer operation is limited into the $10 \mathrm{MHz}$ level with $\Delta V_{\mathrm{a}}<2 \mathrm{kV}$. Some kinds of the FADIS are considered for the NTM suppression in the JT-60SA in this paper. Conditions and targets to be considered are listed in Table 1. 2- $f$ operation is desired to conduct various experiments in the JT-60SA. The operation with arbitrary elliptical polarization states is also expected for ECCD with the oblique injections.

\section{Interferometer and Resonant Ring Diplexers in Circular Corrugated Waveguides}

\subsection{Mach-Zehnder interferometer type}

Transmission lines or launchers can be selected with an interferometer ring diplexer with small frequency control of the gyrotron [11]. Figure 3(a) shows a schematic of a general Mach-Zehnder interferometer in a waveguide line. An inlet port 1 with frequency of $f_{1}$ is used in the diplexer operation. The incident power at the inlet port 1 is divided into transmitted and reflected lines at a splitter, and merged together with at a combiner again. Output power at the outlet port A or B depended on phase difference at the combiner between the transmitted $T$ and reflected $R$ 


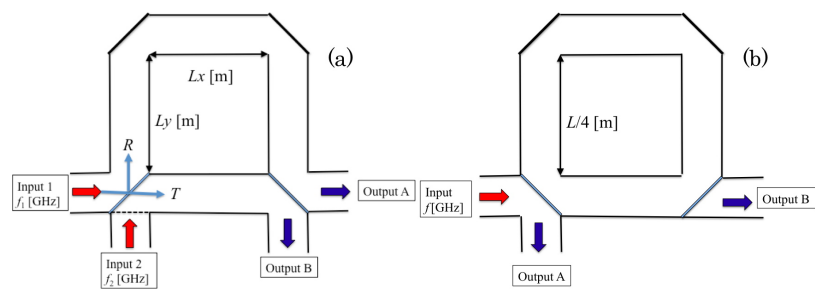

Figure 3. Schematics of (a): a general Mach-Zehnder interferometer in a waveguide line and (b): a resonator type diplexer in a waveguide ring with the round-trip length of $L$.

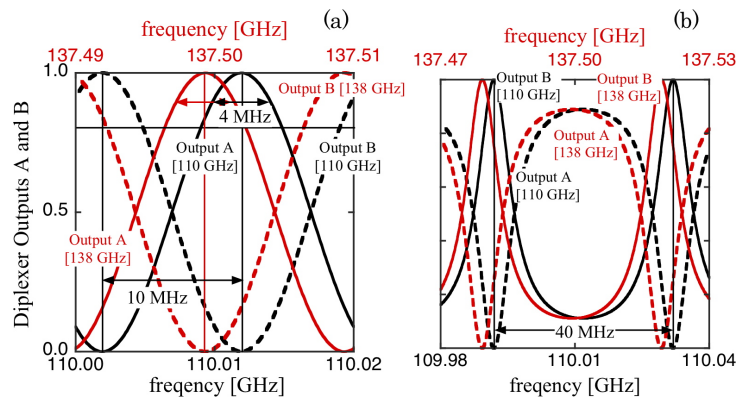

Figure 4. (a): Output power dependences of outlets A and B on operating 110 and $138 \mathrm{GHz}$ frequencies at $L_{\mathrm{y}}=7.5 \mathrm{~m}$ and $R=T=0.5$ at (a): Mach-Zehnder interferometer and (b): ring resonator in circular corrugated waveguide lines.

components after waveguide transmission. Here reflectivity $R$ and transmissivity $T$ were defined as factors on the power. The output powers at the outlet ports $\mathrm{A}$ and $\mathrm{B}, P_{\mathrm{A}}$ and $P_{\mathrm{B}}$, are expressed as follows.

$$
\begin{aligned}
P_{\mathrm{A}} & =R^{2}+T^{2}+2 R T \cos \phi, \\
P_{\mathrm{B}} & =2 R T(1-\cos \phi), \\
\phi & =\phi_{0}+4 \pi L_{\mathrm{y}} / \lambda_{\mathrm{g}},
\end{aligned}
$$

where $\phi_{0}$ is the phase offset at the splitter/combiner and at the $L_{x}$ transmission, and $2 L_{\mathrm{y}}$ is the difference of propagating distances between the $R$ and $T$ components as shown in Fig.3(a). The waveguide wavelength $\lambda_{\mathrm{g}}$ of the main transmission $\left(\mathrm{HE}_{11}\right)$ mode is expressed as $\lambda_{0} / \sqrt{1-\left[\lambda_{0} \chi_{11} /(\pi D)^{2}\right]}$. Here $\lambda_{0}$ and $\chi_{11}$ were wavelength in vacuum and a first zero-crossing solution of 0-th order Bessel function of the first kind $J_{0}$, respectively. The diameter $D$ of the circular corrugated waveguide is 60.3 $\mathrm{mm}$ in the JT-60SA ECHCD system. The frequency shift necessary for switching $\delta f$ between $\phi=0$ and $\pi$ became around $c /\left(4 L_{\mathrm{y}}\right)$, assuming $\lambda_{\mathrm{g}}=\lambda_{0}$ in the oversized corrugated waveguide. Here $c$ is the speed of light. The difference between $\lambda_{\mathrm{g}}$ and $\lambda_{0}$ is comparable in magnitude to $\lambda_{0}\left(\lambda_{0} / D\right)^{2}$. The power $P_{\mathrm{A}}$ is changed from $(R-T)^{2}$ to $(R+T)^{2}$ with $\phi=[0, \pi]$. The specification of the switching frequency step for diplexer operation is $10 \mathrm{MHz}$ as listed in Table 1, and then $L_{\mathrm{y}}$ becomes $7.5 \mathrm{~m}$. Figure 4 (a) shows output power dependences at the outlets $\mathrm{A}$ and $\mathrm{B}$ on the operating 110 and $138 \mathrm{GHz}$ frequencies at $L_{\mathrm{y}}=$ $7.5 \mathrm{~m}$ and $R=T=0.5$. The phase offset $\phi_{0}$ is set to zero for simplicity. The output is switched by the $10 \mathrm{MHz}$ con-

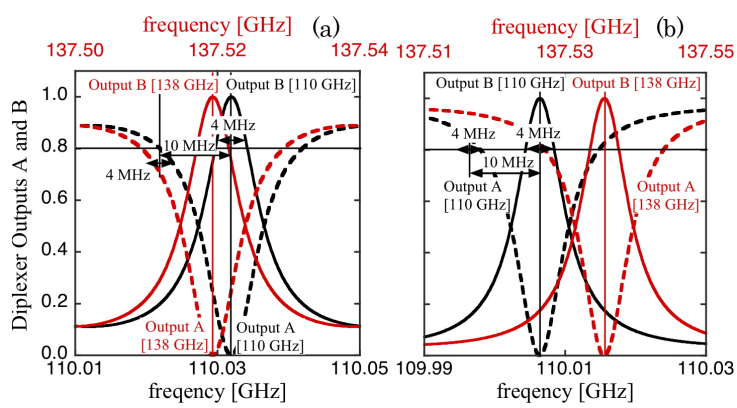

Figure 5. (a) Resonant cavity dependence on operating frequency with $L_{\mathrm{y}}=7.5 \mathrm{~m}$ and $R=T=0.5$. (b) Optimized cavity dependence on operating frequency with $L_{\mathrm{y}}=4.709 \mathrm{~m}, R=0.67$ and $T=0.33$.

trol not depending the operating frequency itself, and more than $80 \%$ components $(\sim 90 \%)$ can be transmitted into the specific outlet ports with the $4 \mathrm{MHz}$ frequency dips. The Mach-Zehnder interferometer diplexer is available for $2-f$ $(110 / 138 \mathrm{GHz})$ application if the incident power was split and combined at $R=T=0.5$. The performance of splitter and combiner for 2- $f$ application is discussed later.

\subsection{Ring Resonator type}

Figure 3(b) shows a schematic of resonator type diplexer in a waveguide ring with the round-trip length of $L$. There are two splitter/combiner plates to construct the resonator ring. Figure 4 (b) shows output power dependence at the outlets A and B on the operating 110 and $138 \mathrm{GHz}$ frequencies at $L_{\mathrm{y}}=7.5 \mathrm{~m}$ and $R=T=0.5$. The output powers at the outlets $B$ and $A$ are expressed as

$$
\begin{aligned}
& P_{\mathrm{B}}=T^{2} /\left[1+R^{2}-2 R \cos \left(\phi_{0}+2 \pi L / \lambda_{\mathrm{g}}\right)\right], \\
& P_{\mathrm{A}}=\left[1-P_{\mathrm{B}}\right],
\end{aligned}
$$

where $\phi_{0}$ is the phase offset at the two splitter/combiner components but is set to zero for simplicity in Fig.4 (b). The power $P_{\mathrm{B}}$ is changed from $T^{2} /\left(1+R^{2}\right)$ to $T^{2} /\left(1-R^{2}\right)$ according to the frequency characteristics of the resonant cavity. Figure 5(a) shows the cavity dependence around resonant frequencies on the operating 110 and $138 \mathrm{GHz}$ at $L_{\mathrm{y}}=7.5 \mathrm{~m}$ and $R=T=0.5$. The period of the resonant cavity dependence on the frequency $\delta f$ is $c / L$ at $\lambda_{\mathrm{g}} \sim \lambda_{0}$. The frequency period $\delta f$ in Fig.5 (b) is $40 \mathrm{MHz}$, but the frequency shift for switching is around $10 \mathrm{MHz}$ in the resonant dependence as shown in the figure. The resonant cavity dependence can be described with $\delta f$, not the operating frequency, as shown in the figure. The transmission line is switched by the $10 \mathrm{MHz}$ control, but it is difficult for more than $80 \%$ power to be transmitted into the specific waveguide line in the presence of frequency dips of $4 \mathrm{MHz}$. The resonant cavity dependence was optimized in $(R, T, L)$ parameters to transmit more than $80 \%$ power to the specific line. The $Q$ value in the resonant cavity is determined by $R$ or $T$ and $L$. Therefore, the slope of the cavity resonance becomes steep and gently by larger $R$ and $L$, respectively. The minimum power at the outlet $\mathrm{B} P_{\mathrm{B}}^{\min }$ 

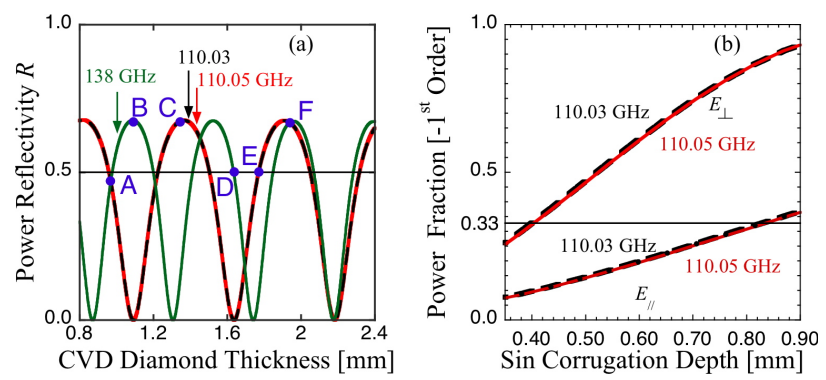

Figure 6. (a) $R$ dependence on CVD-diamond plate thickness in s-polarization at two operating 110 and $138 \mathrm{GHz}$ frequencies. (b) Dependences of -1st order normalized $T$ component on sinusoidal corrugation depth of grating reflectors for linear electric fields $E_{\perp}$ and $E_{/ /}$in perpendicular and in parallel to the corrugation groove at $110 \mathrm{GHz}$.

becomes small by a large $R$ as $P_{\mathrm{B}}^{\min }=T^{2} /\left(1+R^{2}\right)$. Figure 5(b) shows the optimized cavity dependences of the outlets $\mathrm{A}$ and $\mathrm{B}$ around the resonant frequency at $L_{\mathrm{y}}=$ $4.709 \mathrm{~m}, R=0.67$ and $T=0.33$. The reflectivity $R$ had an upper limit $(\sim 0.68)$ on the splitter/combiner performance as mentioned below. Mode filtering effect has been pointed out as another attractive aspect in the resonant ring at the transmission line. The $(R, T, L)$ parameters in the figure are chosen such that the main $\mathrm{HE}_{11}$ mode is resonant; however, the unwanted $\mathrm{HE}_{21}, \mathrm{HE}_{12}$ and $\mathrm{HE}_{13}$ modes are off-resonant due to tiny differences of the waveguide wavelength. Since this issue is outside of the scope in this paper, it will be discussed elsewhere. The transmission line can be switched by the $10 \mathrm{MHz}$ frequency control in both operating frequencies, and more than $80 \%$ power could be transmitted into the specific line with the $4 \mathrm{MHz}$ frequency dips. In principle, the ring resonant diplexer could be applied for the 2- $f(110 / 138 \mathrm{GHz})$ application in the JT-60SA ECHCD system, taking the splitter/combiner performance into account.

\subsection{Splitter and Combiner}

Splitters and combiners were used to construct diplexers of Mach-Zehnder interferometer and resonator ring types. Concepts for CW operation at MW power level have been considered, and an attractive candidate for splitters/combiners is a plate made from CVD-diamond. For dielectric plates, reflectivity $R$ and transmissivity $T$ are determined by the boundary conditions at the surfaces between vacuum/air and the dielectric with refractive index $n$. The $R$ and $T$ components are determined by the refractive index $n$. For incident angles of 45 degree, indices $n$ of 1.84 and 3.34 are required to obtain $R=0.5$ for p- and s- polarizations Note that $R$ increases with $n$ due to the increasing discontinuity of the boundary surface. As $n$ of CVD-diamond is 2.3, a CVD-diamond plate is useful for splitters and combiners only in the s-polarization. Figure 6(a) shows $R$ dependence on the thickness for two operating frequencies in the s-polarization. The dependences for 110.03 and $110.05 \mathrm{GHz}$ including switching frequency component are also shown in the figure. The maximum
$R$ of 0.676 is obtained, indicating good applicability of the CVD-diamond plate for the resonator ring diplexer, too. Some operating points are proposed for the 2- $f$ application here. Points A and F are useful for the MachZehnder interferometer and resonator ring applications in both operating frequencies, respectively. At these points, the diplexers works in the 2- $f$ application. Points B and D are useful for the resonator ring and Mach-Zehnder interferometer applications at $138 \mathrm{GHz}$ only. Since the reflectivity $R$ is close to zero at $110 \mathrm{GHz}$, the power of $110 \mathrm{GHz}$ can be transmitted without change by the diplexer. It is one choice of operating scenarios in the $2-f$ transmission. Fine tuning of the waveguide length for the operating frequency is required in the diplexer circuit, but it will be sometimes difficult for the 2- $f$ application in the rigid waveguide line. The diplexer works for only one frequency with the fine tuning, and the other frequency power could be transmitted with no modification of the transmission line. Points $\mathrm{C}$ and $\mathrm{E}$ are proposed for the diplexer application only at 110 $\mathrm{GHz}$. Although the $\mathrm{CW}$ window has been well developed with the CVD-diamond plate, the CVD-diamond splitter still bears the risk of damage. The operating polarization is limited to s-polarization. The diplexer in circular corrugated waveguides is one of the attractive candidates, but some critical aspects are to be considered.

\section{Ring Resonator type in Quasi-optical Transmission Line}

This diplexer type has been developed well, and its performance has been already demonstrated at the MW power system [6]. The resonant cavity dependence on the frequency is the same as for the ring resonator in the waveguide with identical $(R, T, L)$ parameters, if the scattering coefficients at quasi-optical coupling mirrors were not taken into account. If the quasi-optical diplexer is operated directly in the $\mathrm{HE}_{11}$ mode, the Matching Optics Unit (MOU) to the circular corrugated waveguide line is vey compact, and this kind of the diplexer can be applied for the JT-60SA ECHCD system also. One of the issues to be considered in the quasi-optical diplexer is the design of the splitter and combiner for the JT-60SA application. Grating reflectors have been used as splitters and combiners in this quasi-optical diplexer; here, the 0 -th and -1st order reflection beams on the corrugated surface are employed. The 0 -th and -1st order components correspond to the $R$ and $T$ components in the ring resonator. Figure 6(b) shows dependences of -1 st order $T$ components normalized by the $(R+T)$ components on sinusoidal corrugation depth for linear electric fields $E_{\perp}$ and $E_{\|}$in perpendicular and in parallel to the corrugation groove at $110 \mathrm{GHz}$. This grating performance was evaluated by a 3-dimensional electromagnetic solver of MoM ( Method of Moment ) [12]. A $T$ component of 0.33 is required for the desired transmission function of the resonant ring diplexer. Sinusoidal grating splitters and combiners can be designed for both polarizations, however, for equal splitting ratio the corrugation depth is different. Grating splitters and combiners for arbitrary incident polarization states require very 


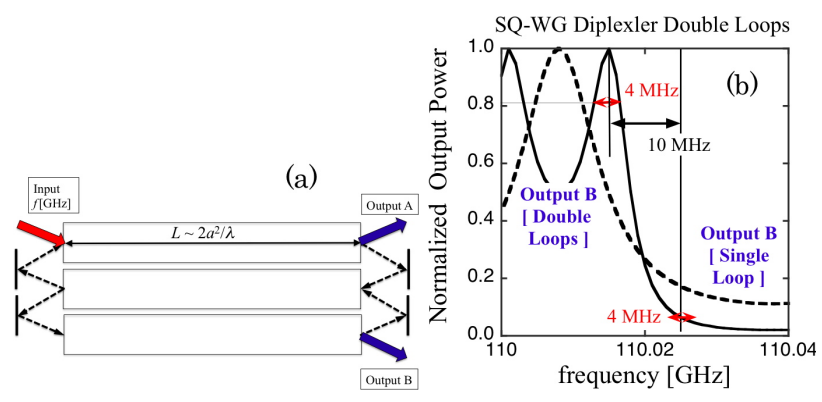

Figure 7. (a) Schematic of double loop cavity system with the square corrugated waveguide splitter/combiner. (b) Resonant cavity dependences of single- and double-loop square corrugated waveguide diplexers at $L_{\mathrm{SCW}}=2.1 \mathrm{~m}$.

specific corrugation profiles. The grating performance is affected by the incident wave vector and the corrugation pitch. The $T$ component of the grating reflection is 30 $\mathrm{dB}$ down at $138 \mathrm{GHz}$. One-frequency operation scenario might be proposed in the quasi-optical diplexer with no modification of the transmission line for two frequencies, but diffraction or coupling losses at quasi-optical mirrors in the MOU might be increased for the non-operating frequency of the diplexer.

\section{Square Corrugated Waveguide Diplexer}

Square corrugated waveguide launchers based on the remote steering concept were proposed for the upper port launchers in ITER. There are two kinds of remote steering launchers. One is the symmetric directional launcher, whose launching angle is identical to the input angle, and the other is the anti-symmetric with the opposite directional launching angle as the input; these features depend on launcher length $L_{\mathrm{SCW}}$ and side of square aperture $a$. In the traditional concept, operational parameters of $L_{\mathrm{SCW}}$ and $a$ are described as $L_{\mathrm{SCW}}=8 a^{2} / \lambda_{0}$ and $4 a^{2} / \lambda_{0}$ for symmetric and anti-symmetric directional launchers, respectively. A square corrugated waveguide of $L_{\mathrm{SCW}}=2 a^{2} / \lambda_{0}$ can be used as a splitter of an input beam into symmetric and anti-symmetric directions. In the JT-60SA ECHCD application, the side $a$ of the square aperture was selected to be $0.0535 \mathrm{~m}$ such that the cross-section area of $a^{2}$ was the same as $\pi D^{2} / 4$ in the circular corrugated waveguide. Since $L_{\mathrm{SCW}}$ is determined by $L_{\mathrm{SCW}}=2 a^{2} / \lambda_{0}$, the propagating length of the diplexer is not a free parameter to consider switching properties on the operating frequency. Moreover, allocation of $R$ and $T$ power fractions is difficult in the square corrugated waveguide splitter. The $Q$ value of the cavity resonant dependence cannot be adjusted by $(R, T, L)$ parameters like the other resonant ring diplexer. Therefore, a double loop resonant cavity system has been proposed to obtain high- $Q$ diplexer performance $[13,14]$. Figure 7 (a) shows a schematic of the double loop cavity system with the square corrugated waveguide splitter/combiner. The output power at outlets A and B can be

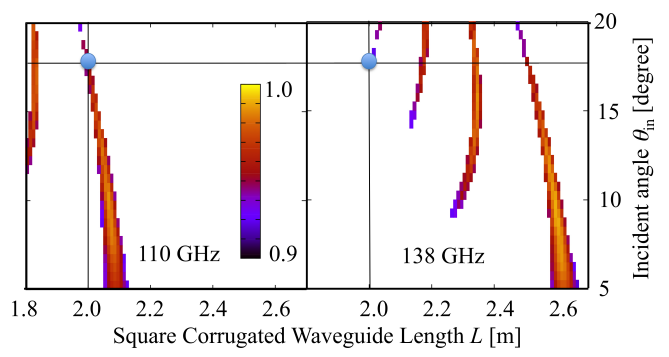

Figure 8. Contour plot of matching coefficients $(>0.9)$ between two electric field distributions of an ideal beam and of the beams excited at the outlet aperture and split into the symmetric and anti-symmetric directions, as a function of $L_{\mathrm{SCW}}$ and incident angle $\theta_{\text {in }}$ for 2- $f(110 / 138 \mathrm{GHz})$ operations. The side $a$ of the square aperture was $0.0535 \mathrm{~m}$.

expressed as

$$
\begin{aligned}
P_{\mathrm{A}} & =\frac{\left(3-4 \cos \phi_{\text {total }}\right)^{2}}{\left(18-24 \cos \phi_{\text {total }}+8 \cos 2 \phi_{\text {total }}\right)} \\
P_{\mathrm{B}} & =\frac{1}{\left(18-24 \cos \phi_{\text {total }}+8 \cos 2 \phi_{\text {total }}\right)}
\end{aligned}
$$

where $\phi_{\text {total }}$ is the total phase delay of the round-trip in one resonant ring including the quasi-optical (free-space) path delay. Figure 7(b) shows the resonant cavity dependences of single- and double-loop square corrugated waveguide diplexers with $L_{\mathrm{SCW}}=2.1 \mathrm{~m}$ for the $110 \mathrm{GHz}$ operation. The distance between the waveguide-end and the quasi-optical mirror was $0.02 \mathrm{~m}$. The slope of the resonance in the double loop diplexer is sufficiently steep to switch the outputs by a $10 \mathrm{MHz}$ frequency shift, with the frequency dips of $4 \mathrm{MHz}$. Therefore, square corrugated waveguide is useful for diplexer applications, but the 2- $f$ operation is impossible in the traditional splitter concept of $L_{\mathrm{SCW}}=2 a^{2} / \lambda_{0}$. Extended operational regions were proposed for the remote steering launcher in ECHCD experiments on the TRIAM tokamak [15], based on Matching Coefficient (MC) analysis. A symmetric directional launcher with extended launching angle was successfully developed and utilized for the high power $(\sim 200 \mathrm{~kW})$ application on the TRIAM tokamak. Extended operation regions for a diplexer have been surveyed using a similar approach as for the symmetric remote steering launcher design with the extended angle. Figure 8 shows a contour plot of $\mathrm{MC}(>0.9)$ between two electric field distributions of an ideal beam and the beams excited at the outlet aperture, as a function of $L_{\mathrm{SCW}}$ and incident angle $\theta_{\text {in }}$ for 2- $f(110 / 138 \mathrm{GHz})$ operations. The side $a$ of the square aperture was $0.0535 \mathrm{~m}$. High MC operational region is found for the normal and the 3rd extended branches at $L_{\mathrm{SCW}}=2.0 \mathrm{~m}$ and $\theta_{\text {in }}=17.5$ degree for 110 and $138 \mathrm{GHz}$, respectively. Figure 9 shows electric-field intensity and phase distributions, $I(x)$ and $\psi(x)$, radiated from the square corrugated waveguide at 110 and $138 \mathrm{GHz}$ in the normal and 3rd operational branch operations, respectively. The radiation fields were evaluated by the developed Kirchhoff integral code [16] at the propagation distance of $z=0.2 \mathrm{~m}$. The beams were obliquely injected at $\theta_{\text {in }}=17.5$ degree in 


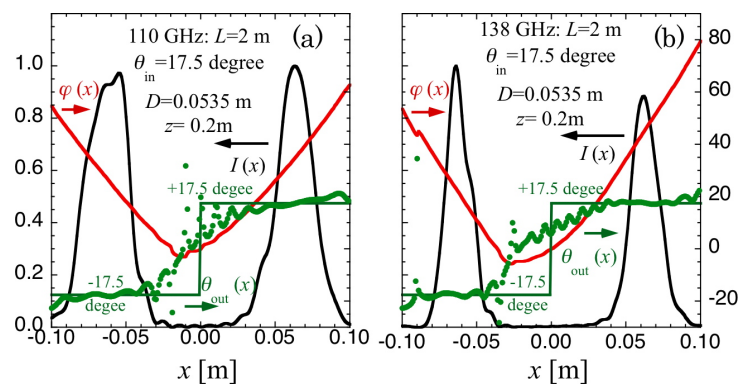

Figure 9. Electric-field intensity and phase distributions, $I(x)$ and $\psi(x)$, radiated from the square corrugated waveguide in the normal and 3rd operational branches at 110 and $138 \mathrm{GHz}$, respectively. Local output angle profile $\theta_{\text {out }}(x)$ in the $x$ direction determined by $\tan ^{-1}\left[k_{x}(x) / k_{0}\right]$ is also shown in the figure. Here, $k_{x}$ and $k_{0}$ are wavenumbers in the $x$ direction and in magnitude in free-space propagation.

the $x-z$ plane. The phase distribution $\psi(x)$ was $k_{x} x$ profile, where $k_{x}$ was a wavenumber component in the $x$ direction. The $k_{x}$ profile, which was the derivative of $\psi(x)$ on $x$, showed the propagating direction in the $x$ coordinate. Local output angle profile $\theta_{\text {out }}(x)$ in the $x$ direction determined by $\tan ^{-1}\left[k_{x}(x) / k_{0}\right]$ is also shown in the figure. Here, $k_{0}$ is the wavenumber in free-space propagation. The main lobes of the two split beams were properly obtained at the output angle of 17.5 degree in response to the incident angle at both 110 and $138 \mathrm{GHz}$ frequencies. The features shown above do not depend on polarization; therefore, a diplexer based on square corrugated waveguide can be operated at arbitrarily polarized radiation. Since Ohmic loss is high in waveguides with rectangular corrugation at large $\theta_{\text {in }}$ and as it depends on the incident polarization, square corrugated waveguides with bell-shaped corrugation have been considered to reduce the Ohmic loss [17]. The Ohmic loss properties were experimentally confirmed with a Fabry-Perot cavity technique [18]. Coupling mirror surfaces should be individually prepared for each operating frequency to reduce the diffraction losses in the couplings into the square corrugated waveguide. A mirror adjuster system will be considered to change the mirror surfaces prepared at both faces of the mirror, and also to align mirror position and angle corresponding to the split beams radiated from the waveguide in both operating frequencies. Fine tuning of $\psi_{\text {total }}$ is possible by the mirror position and angle control for each operating frequency. However, in general the phase delays in the waveguide are different between two orthogonal electric fields perpendicular and parallel to the corrugation grooves. For a specific operating frequency, the bell-shaped corrugation can be optimized to compensate this phase difference, as was experimentally confirmed using the Fabry-Perot cavity technique [18]. For the 2- $f$ application, a possibility to bridge the phase difference between the two orthogonal fields is an additional corrugation of the coupling mirror surfaces. The square corrugated waveguide FADIS does not have fragile components with risk of damage like the CVD-diamond splitter/combiner, and in principle allows operation at arbitrary polarization. Therefore, the square corrugated waveguide FADIS with 2 resonant rings is one of most attractive candidates for the JT-60SA ECHCD system requiring the specifications in Table 1.

\section{Summary}

Various types of the FADIS have been considered for the 2- $f$ application for the JT-60SA ECHCD system. MachZehnder interferometer type and ring resonator type in circular corrugated waveguide could be attractive candidates, but operation is limited to linear polarization, and there is a risk of damage if CVD-diamond splitters/combiners will be adopted as proven pieces for $\mathrm{CW}$ operation at $\mathrm{MW}$ power level. The quasi-optical ring resonator type does not work in the 2- $f$ application because of the dispersion of the grating splitters. The square corrugated waveguide FADIS with 2 resonant rings is most attractive because there is no risk to damage fragile parts like CVD-diamond disks, and no limitation of the operating polarization in principle. A bell-shaped corrugation profile is considered to reduce the Ohmic loss in the square corrugated waveguide and to construct the resonator ring at small phase difference in the 2- $f$ application for arbitrary polarization states.

\section{References}

[1] A. Isayama, et al., Nucl. Fusion, 431272 (2003).

[2] K. Nagasaki, et al., Nucl. Fusion, 43 L7 (2003).

[3] A. Isayama, et al., Nucl. Fusion, 490550006 (2009).

[4] M. Maraschek, et al., Phys. Rev. Lett., 98025005 (2009).

[5] R. Prater, et al., Nucl. Fusion, 47371 (2007).

[6] W. Kasparek, et al., Nucl. Fusion, 48054010 (2008).

[7] T. Kobayashi, et al., Trans. Fusion Sci. Technol. 63, $1 \mathrm{~T} 160$ (2013).

[8] A. Isayama, et al., Plasma and Fusion Res., 7 , 2405029 (2012).

[9] N. Doelman, et al., EPJ Web of Conferences 32, 04005 (2012).

[10] T. Kobayashi, et al., Nucl. Fusion, 51103037 (2011).

[11] W. Wubie, et al., Proc. 33rd Int. Conf. Infrared, Millimeter, and Terahertz Waves (IRMMW-THz 2008), Pasadena, California, September 2008.

[12] http://www.wipl-d.com/products/pro9.php.

[13] A. Bruschi, et al., Fusion Sci. Technol., 53, 97 (2008).

[14] W. Kasparek et al., Fusion Sci Technol., 59729 (2011).

[15] H. Idei, et al., Nucl. Fusion 46, 489 (2006).

[16] H. Idei, et al., J. Plasma Fusion Res., SERIES, 9112 (2010).

[17] B. Plaum et al., J. Infrared Milli. Terahz. Waves, 32 482 (2011).

[18] W. Kasparek, et al., Proc. of 6th European Conf. on Antennas and Propagation (EU-CAP), Prague, 2012. talk CM01.6, paper 1569533861. 